\title{
The Diagnosis of Multiple Personality Disorder
}

Joshua D. McDavid, MD, MPH

University of Pittsburgh

Follow this and additional works at: https://jdc.jefferson.edu/jeffjpsychiatry

Part of the Psychiatry Commons

Let us know how access to this document benefits you

\section{Recommended Citation}

McDavid, MD, MPH, Joshua D. (1994) "The Diagnosis of Multiple Personality Disorder," Jefferson Journal of Psychiatry. Vol. 12 : Iss. 1 , Article 7.

DOI: https://doi.org/10.29046/JJP.012.1.004

Available at: https://jdc.jefferson.edu/jeffjpsychiatry/vol12/iss1/7

This Article is brought to you for free and open access by the Jefferson Digital Commons. The Jefferson Digital Commons is a service of Thomas Jefferson University's Center for Teaching and Learning (CTL). The Commons is a showcase for Jefferson books and journals, peer-reviewed scholarly publications, unique historical collections from the University archives, and teaching tools. The Jefferson Digital Commons allows researchers and interested readers anywhere in the world to learn about and keep up to date with Jefferson scholarship. This article has been accepted for inclusion in Jefferson Journal of Psychiatry by an authorized administrator of the Jefferson Digital Commons. For more information, please contact: JeffersonDigitalCommons@jefferson.edu. 


\title{
The Diagnosis of Multiple Personality Disorder
}

\author{
Joshua D. McDavid, M.D., M.P.H.
}

\begin{abstract}
This paper represents an attempt to facilitate the clinician or psychiatrist in making a diagnosis of Multiple Personality Disorder, the most severe of the dissociative disorders. Emphasis will be placed on detecting signs, symptoms, and information that occur within the constituent parts of a typical psychiatric interview, and that are highly suggestive of MPD even in the absence of alter presentation.
\end{abstract}

Although the frequency of the diagnosis of Multiple Personality Disorder (MPD) has increased in the last several years psychiatric residency training programs still give little more than cursory attention to this area $(1,2)$, and as a result, most psychiatrists are not trained in systematic assessment of the dissociative disorders.

MPD is best conceptualized as both a complex, chronic dissociative disorder characterized by disturbance of identity and memory (3) and as a post-traumatic condition initiating from abuse or traumatic childhood experiences (4).

It has been hypothesized that dissociative states develop as protection against overwhelming negative stimuli. Given a necessary biological diathesis, the potential for autohypnosis or dissociation, the young, growing, child may form discrete and variably "disconnected" states when confronted with overwhelming trauma. These states are perpetuated by continuing abusive or neglecting relationships, particularly in the absence of restorative experiences with a significant other or others (5). Initially as a kind of "successful" adaptation, the dissociative process insures or increases the likelihood of the child's survival. In other dimensions of the survivor's life, and especially in adulthood, the adaptability becomes a disability, impairing the development of a uniform self-concept and attenuating the formation of stable interpersonal relationships.

The importance of making the diagnosis of MPD is more than just an academic exercise. Failure to make the diagnosis results in substantial morbidity and even death. Patients who leave treatment prematurely do not cease having MPD nor does MPD remit spontaneously (3). Though the treatment can be arduous, the potential for resolution of symptoms and improvement of functioning is real. It is not

Joshua D. McDavid, M.D., M.P.H. is an NIMH Clinical Research Fellow at the Western Psychiatric Institute and Clinic of the University of Pittsburgh. 
uncommon for patients with MPD to have been previously diagnosed with schizophrenia and treated as such with high dose neuroleptics and adjuvants; occasionally an MPD patient will be detected in a chronic care facility or even the back wards of a state hospital (6).

The purpose of this paper is to aid clinicians and psychiatrists in detecting, primarily in adults, MPD, the most clinically complex and dramatic of the dissociative disorders. Although the diagnosis of MPD requires the identification of alternate personalities (alters) this paper will highlight those areas within a typical initial psychiatric interview that lend themselves to discovery of MPD in the absence of alter presentation by identifying the symptoms constituting the polysymptomatic constellation frequently associated with MPD.

\section{DIAGNOSTIC CRITERIA}

Besides "the presence of other personalities" the DSM-III-R criteria have not offered much in the way of guidelines for diagnosing MPD (7). It has not addressed the post-traumatic nature of the disorder, and the only operationalized criterion is one dissociative symptom, personality change. Because of the paucity of criteria articulated in the DSM-III-R, clinicians have not learned to inquire in a way that will extricate MPD from other syndromes.

Although the diagnosis requires the identification of distinct personalities, or alters, MPD consists of a constellation of non-specific signs and symptoms (polysymptomatology) and is relatively pleiomorphic $(8,9,10,11)$. In classic MPD as defined by DSM-III-R, each personality state, as a distinct entity, takes full control of the person's behavior. Classic MPD is probably not as common as less dramatic presentations with less than full control and distinctness $(8,12)$. Various authors have attempted to conceptualize the subtle gradations, calling them atypical variants, ego state changes, co-presences, isomorphisms, and fragments $(8,11,12,13)$.

DSM-IV changes the name of MPD to Dissociative Identity Disorder, and it now eliminates the requirement of "full" control as part of the A criterion. It introduces the requirement of amnesia as a criterion for the diagnosis since evidence suggests that it is a symptom in $90 \%$ of patients with the disorder. However, whether MPD patients experience formal amnesia remains controversial. A new criterion for the DSM-IV is: "The inability to recall important personal information that is too extensive to be explained by ordinary forgetfulness" $(14,15,16)$. A fourth criterion stating that "the disturbance is not due to a Substance-Induced Disorder" has also been introduced (16).

The DSM is not alone in minimally demarcating accompanying signs and symptoms of MPD. In the new International Classification of Diseases, or ICD-10, MPD has not been listed formally as a diagnostic entry (17), but is subsumed under "other dissociative disorders" (18). 


\section{CLINICAL PRESENTATION}

\section{General Considerations}

The concept of "windows of diagnosability" has been applied to MPD because of its covertness and the tendency of patients with the disorder to dissimulate (5). Age, gender, developmental history, subjective experiences, previous experiences with mental health workers, and therapist expertise will all influence the expression of symptoms during an interview. For example, whereas young children may be the most susceptible and least resistant to early recognition of the disorder, their dissociative symptoms appear more vague and frequently seem to have other possible diagnoses $(15,19,20)$. Adolescents seem to be the most complex and diverse of patients with MPD and least likely to reveal alter personalities (15).

Kluft $(8,15)$ reports that among adults $20 \%$ of those with MPD resist detection; $50 \%$ have sustained periods of symptom quiescence lasting sometimes a year or more; and only a small minority, $6 \%$, are exhibitionistic about the disorder. In the context of a rising suspicion for the diagnosis, he recommends an extended interview, since spontaneous symptoms of dissociation may eventually emerge with fatigue and stress. Nevertheless, the diagnosis frequently requires patience on the part of the clinician. Proper diagnosis during the first interview is the exception, and diagnosis may require weeks, if not months, to determine.

If MPD is suspected or a polysymptomatic presentation occurs, most experienced clinicians recommend that questions pertaining to the presence of alters be relegated to the end of the session once some rapport has been established $(1,9)$, in order not to frighten a patient who often will be revealing the multiplicity for the first time to another. These patients often suffer a profound sense of personal confusion, inferiority, and worthlessness and view the world as frightening, overwhelming, and unsympathetic. Revelation of their internal disorganization to others causes great distress (4). Reluctance to reveal the self system also may resonate with the childhood fear of being threatened or blamed if they reveal the abuse or of being accused as liars (11). In the more classic form of MPD the host or primary personality may not even be aware of the presence of alters and will deny their existence, and such a revelation to the host can in itself be traumatic and frightening.

If the patient responds to probing questions in a puzzling or out of character way, asking if another "personality" is present is the proper intervention (15). A negative response, however, may reflect denial and not necessarily the absence of MPD. A positive response suggests further inquiry given adequate time and recognition that the disclosure could be de-stabilizing and frightening to the patient.

If a polysymptomatic presentation occurs that is suggestive of MPD then the clinician can go farther and ask questions about the presence of other "personalities." The presence of alters usually settles the diagnosis unless there is suspicion of a malingering or factitious patient. Alters are more likely to emerge spontaneously when the patient is in crisis or if the diagnosis has been made previously (9). 
The following questions about other alters can be directly asked: Have you ever been told by others that you seem like a different person? Have you ever referred to yourself by different names? Or, have you ever acted in a completely different manner?

A caveat to asking questions about MPD is the real concern about iatrogenesis, suggesting or even encouraging the formation of alternate personalities. Although there is some evidence that iatrogenesis can occur (3), particularly as a result of hypnosis, the clinician must guard against being too aggressive as well as being too passive in the questioning. Instead of asking patients if they have other personalities or names, asking about whether they have other aspects or parts with contradictory or seemingly unknown affects and experiences may help to guard against this dilemma.

\section{THE PSYCHIATRIC INTERVIEW}

In spite of the lack of guidelines for diagnosing MPD there are several broad factors that suggest MPD; these factors derive from the natural loci of the psychiatric interview though they may emerge during any phase of therapy. These constituents of the interview include the history of present illness, psychiatric, medical, social and developmental history, signs and symptoms, and the mental status exam.

Loewenstein (1) has devised the "symptom cluster method" for assessing MPD and Dissociative Disorders. He divides MPD symptoms into six different phenomenological categories. Process symptoms (i.e. switching), core aspects of a patient's multiplicity, refer to those symptoms that reflect alter transitions or interactions between alters. The other clusters are more familiar, though not necessarily attributed to MPD by clinicians unfamiliar with MPD symptom expression. They include amnesia, autohypnotic, post-traumatic, somatoform, and affective symptoms.

This paper will emphasize those aspects and symptoms of MPD that fall within the framework of a traditional psychiatric interview (see table 1).

Usually the clinician or psychiatrist will not have received any advance direct indication that a patient may have MPD. A composite patient profile that frequently characterizes the presentation of a "typical" MPD patient is as follows:

A 30 year old depressed female with symptoms of amnesia, recurrent suicidal ideation, self destructiveness, a history of sexual abuse, many psychiatric diagnoses and failed treatments.

Such a profile is unusual in uncomplicated affective and anxiety disorders and psychosis. Some patients with personality disorders, particularly borderline personality disorder or a "hysterical" personality style can approach the composite, and such a profile warrants consideration of and investigation into the possibility of MPD.

\section{Patient Identification and History of Present Illness}

The signs and symptoms of the composite which justify particular attention include amnesia without obvious organic explanation, current or past suicidal or 
TABLE 1.

Factors Suggesting MPD that are Part of a Typical Psychiatric Interview

History of Present Illness

Suicide attempts

Self-mutilation of Self-destruction

"Desperate Depression" or Atypical affective symptoms

Symptoms

Amnesia

Fugue

Auditory hallucinations

Schneiderian symptoms

PTSD Symptoms (detachment, avoidance, reexperiencing of trauma, nightmares)

Concurrent somatic and psychiatric symptoms

"Hysteria"

Psychiatric History

Numerous previous diagnosis and treatment failures Prior or Concurrent Disorder:

PTSD

Borderline personality disorder

Eating disorders

Psychotic disorders unresponsive to medications

Somatoform disorder

Substance or Alcohol abuse

Gender identity disorder

Transsexualism or Transvestism

Medical History

Headaches

Numerous physical complaints of sexual nature

Unexplained pain, particularly gynecologic or gastrointestinal

Conversion phenomenon

Fear of physical exams or rejection of care

Family History

Chaotic family situation

Social and Developmental History

Sexual, psychological and physical abuse, especially repetitive and from an early age

History of neglect

Grossly distorted upbringing and impoverished social network in the absence of psychosis

Cult involvement as a young child

Ego-dystonic sexual impulses and acting out

Mental Status Exam

Appearance

Signs of self-injury

Behaviors

Intra-interview amnesia

Spells

Spontaneous regression

Catatonia

Odd behavior despite an apparent relatedness to the interviewer

The use of "we"

Spontaneous voice or accent changes

Sudden involuntary movements

Changes in facial musculature

Changes in handedness 


\section{TABLE 1.}

Factors Suggesting MPD that are Part of a Typical Psychiatric Interview (Cont.)

Fluctuations in creative abilities or styles

Handwriting changes

Affect and Mood

Dramatic shifts in anxiety or mood

Thought Process and content

Pseudodelusions

Abnormal self-concept

Abnormal body concept or image

Obsessive ideation

Marked phobias

Perceptions

Pseudohallucination

Negative hallucinations

Schneiderian symptoms

Illusions, flashbacks, revivifications

Depersonalization/derealization/marked detachment

Cognition

Psychogenic amnesia

Abstraction despite apparent psychosis

self-destructive behavior, a history of sexual or physical abuse, and extensive psychiatric history. A symptom profile which includes somatic complaints with concomitant psychiatric symptoms, or a profile suggesting Post-Traumatic Stress Disorder (PTSD) with detachment, psychic numbing, avoidance, increased arousal, and flashbacks, deserves attention. Extreme negative self-evaluation, disturbance of interpersonal relationships, compulsive sexuality, manipulativeness, reenactments, and adversariality are also frequent epiphenomenon of MPD and severe post sexual abuse trauma $(21,22)$.

\section{Psychiatric History}

The psychiatric history can offer valuable information for considering MPD. The two most important indicators are a history of many prior diagnoses and multiple treatment failures. Numerous psychiatric hospitalizations, usually greater than three, and refractoriness to conventional treatment also may reflect undiagnosed MPD.

A previous diagnosis of PTSD, borderline personality disorder, gender identity disorder, intractable substance or alcohol abuse, schizoaffective disorder, or schizophrenia, especially in a patient who appears to relate well interpersonally, does not respond to treatment, and has a history of sexual abuse should prompt the clinician to consider MPD in the differential diagnosis. 


\section{Medical History}

Numerous physical complaints often accompany MPD. The most common complaint is headaches. Gastrointestinal and sexual symptoms, conversion phenomenon, and unexplained pain syndromes also are quite common (9). Coons (6) reported that $50 \%$ of his patients with MPD were observed with hysterical conversion reactions. Not uncommonly patients will reexperience the physical sensations that some mistreatment had instilled without any conscious awareness of their origin (3).

These patients often have chronic medical illnesses, and they will often not pursue or resist proper treatment. Many of these patients will often fear physical exams and are prone to vasovagal episodes and panic attacks either in relation to the exam or venipuncture. The clinician should question any patient who demonstrates a marked reluctance or dread of a physical exam.

\section{Family History}

Though very non-specific, a present or past chaotic family situation is the most suggestive indicator of MPD within the family history. It is not uncommon for these patients to have families with impoverished relationships and communication networks. Heavy and frequent substance abuse, a criminal record, sexual, emotional, and physical abuse, infidelity, divorce, multi-agency involvement, and unemployment may all be manifestations of the chaos. Conversely, descriptions of "the perfect family" that seem too good to be believable raise suspicion (23).

\section{Social and Developmental History}

Patients with MPD invariably have had a grossly distorted upbringing, generally without adequate or consistent support. Interviews should be sensitive to less commonly identified traumatic experiences such as long confinements, excessive enemas, and medical procedures occurring at an early age. Kluft (15) hypothesizes that most of these patients while growing up had no one with whom to secure a nurturing bond. Questions concerning abuse and neglect are mandatory parts of any psychiatric evaluation. The presence of physical or sexual abuse invites inquiry into the presence of MPD and conversely, the presence of MPD demands inquiry into trauma. Attentive observation of patients while inquiring about abuse history is very important since many patients with MPD or an abuse history will "give themselves away" when asked about these areas. It is not uncommon to get strange or unusual answers to the questions or to see increasing anxiety, a shudder, tremor, or a startle when discussing these areas. Less commonly, dissociative phenomena such as switching, micro-amnesia, or even spontaneous alter emergence occurs. It is best not to ask about abuse directly as this elicits unwarranted defensiveness, but an indirect inquiry, like, "How was discipline handled in your family? How were you punished? What was the worst thing that ever happened to you in childhood?" may elicit more germane information. The evaluator should consider alternative questions and less direct questions if a negative answer suggests denial. Detailed questions about abuse 
can and should be deferred until a more trusting and sustained relationship develops. It is important to mention that during therapy once a therapeutic alliance has been established questions about abuse deserve repeated inquiry even if initial negative responses seemed appropriate. Patients deny abuse for many reasons: fear, amnesia, or because of misunderstanding about the definition of abuse.

Finally, analogous to the dilemma discussed above about asking patients if they have separate identities and iatrogenically causing MPD is the concern about "coaching" a patient into admitting, falsely or exaggerating, a history of abuse. Although almost too obvious to state, coercion and even suggestion have no place in a psychiatric interview. As important as an abuse history may be to a patient's psychology and behavior it should not be forgotten that a history of abuse does not by itself imply pathology or MPD, and abuse should not be the therapist's primary focus or agenda without considering other factors such as presenting complaint, interpersonal relationships and behavior, and the patient's own perception of the issues that require focus. It may be impossible to prevent or immediately detect the patient who deceives, exaggerates, or distorts but the problem can be reduced in the context of a sensitive and non-judgmental evaluation, by a clinician who can call on experience and judgment.

\section{Mental Status Exam}

Appearance: As a general rule during the first interview MPD patients do not appear unusual or different from the great majority of patients. There is a subset that may appear odd or wear mismatched or inappropriate clothes. On occasion the clinician will observe overt signs of self-injury.

Over subsequent sessions curious changes in appearance may be recognized: significantly different styles of clothing, hair, makeup, glasses, posture, and jewelry. Most of these changes are subtle and not dramatic so clinical sensitivity to such changes will increase the likelihood of recognition (1).

Behaviors: Facial changes, body shifts, voice tones, accents, change in handedness, eye changes, involuntary movements, and the use of "we" are less subtle signs, and are more likely to be captured during initial interviews. Even so, an initial interview without such behavior is common. Pertinent behavioral manifestations can occur any time during the interview: intra-interview amnesia, marked or spontaneous regression, spells, catatonia, and hysteria. These should be suggestive clues that warrant specific questions about blackouts, time loss, dissociation and the existence of alters. In the event of the appearance of an alter, the focus on the diagnosis becomes predominant and the reasons for emergence should be explored.

Affect and Mood: As mentioned, a frequent presentation of MPD is in the context of an affective illness or anxiety disorder. Though affect and mood changes are non-specific, they tend to be exaggerated. Dramatic shifts in affect during the interview or between sessions can reflect switching behavior. Increasing anxiety in relation to questions concerning non-specific symptoms or about MPD directly should raise the suspicion of the diagnosis. 
Thought Process: In the context of a crisis presentation, a patient's stream of thought can be markedly loose, rapid, illogical, and without goal directedness. Such thought processes can represent rapid alter switching or conflicted "internal dialogue" among alters that "spill out" $(8,9)$. At baseline or not in crisis, MPD patients do not have a formal thought disorder or thought process dysfunction.

Abnormal thought contents (pseudodelusions, phobias, obsessions, abnormal self-concepts) do occur and are more likely to persist over time. Under usual conditions MPD patients do not suffer or demonstrate psychotic ideation. Under severe stress they may display "micro-psychotic episodes" similar to some personality disorders. However, even during periods of relative quiescence these patients often display symptoms that closely resemble psychotic ideation. Three of the more common pseudodelusions are the belief that alters are separate entities, that one alter can do harm to another without suffering consequences, and that one is being controlled. Often once the dynamics of the delusions are understood what appears as a delusion usually is a reflection of internal conflict among alters, particularly in relation to the host or primary personality (9). Further, these delusions rarely present as a belief that an external agency is persecuting them or sending them messages through the media, symptoms more commonly seen in true psychotic illness.

Similarly, obsessive or compulsive ideation or behavior can manifest in MPD and may reflect alters in conflict, "canceling out" each others' thoughts or behaviors or inserting intrusive thoughts (8).

These patients often have significant phobias, particularly in relation to themes of previous trauma. Certain social situations, words, objects, or emotional states can cue switching behavior that may manifest as phobic behavior (9).

Perception: It is not at all unusual for MPD patients to report perceptual disturbances: hallucinations (better characterized as pseudohallucinations), negative hallucinations, illusions, out of body experiences, and depersonalization and derealization. If the clinician accepts such endorsement without further inquiry, it is easy to confuse the symptoms with true psychotic illness. Other symptoms, including severe regressive behavior and flashbacks or revivifications, can be easily confused with catatonia and visual hallucinations, respectively.

Schneiderian first-rank symptoms are often reported, e.g., made phenomenon: made impulses, feelings, and volitional acts (24). Patients with MPD often describe hearing voices arguing and making comments about their thoughts (25). These symptoms are better understood as pseudohallucinations than true loss of reality testing; passive influence phenomenon and internal voices may represent covert conflict among alters for control. If amnestic barriers between alters become more fluid the likelihood of first-rank symptoms may diminish $(8,9)$.

The following approach to auditory hallucinations should be taken to dissect true psychosis from MPD. Inquiry should focus on whether the voices reside in the patient's head and whether the voices will speak with the clinician. Only infrequently, about $20 \%$ of MPD patients report auditory hallucinations outside of their heads (8). Asking the patient if it is possible to talk to his or her voices can lead to emergence of 
alters or give the clinician some sense of the depth of psychosis. Voices that are chaotic, incoherent, or irrational probably represent manifestations of psychosis whereas alter voices, though dramatic, can be understood through dialogue (10). Truly psychotic voices respond to medication and will become quiescent for periods of time. The voices of patients with MPD persist and generally do not respond to medication $(9,15)$.

The so called "negative symptoms" of schizophrenia usually do not characterize MPD (6); however, oddness and bizarreness can be attributed to some alter personalities. Generally, MPD patients relate interpersonally much better than patients with schizophrenia. Perceptual disturbances such as illusions, visions, flashbacks and revivifications can occur during initial evaluations or as principle motivations for seeking psychiatric care if memories, external cues, or stimuli rekindle images of trauma.

Cognitive Exam: The constituents of the cognitive exam-attention, language, praxis, visuospatial functions, calculations, and abstract reasoning-are generally intact in patients with MPD (9). Characterization of memory impairment in these patients is complex. The type of amnesia they suffer is psychogenic and frequently characterized by asymmetry; the primary personality is unaware of alters but not vice versa (4). Generally, short term memory and memory for skills, information, facts, concepts, and vocabulary, the so called implicit semantic memory, remain intact (26), but there are notable exceptions (27), particularly across alters with rigid amnestic barriers. Discrete gaps in long term memory, particularly involving autobiographical material and childhood events, is common. It is important to remember that memory deficits in patients with MPD are not generally structural, may be "state dependent" to the trauma, and are potentially reversible, resolving when the antecedents to the amnesia are abreacted or understood (28).

\section{IMPRESSIONS ON THE EVALUATOR}

Clinicians unaccustomed to treating or evaluating MPD may find these patients perplexing, strange, bizarre, or even frightening. It takes time to get over the initial fascination or negative reactions that may develop. Since many of these patients present in crisis the initial contact can be quite intense. It is not unusual for clinicians to experience some of the dissociative phenomenon that the patient does, for example, mild intra-interview amnesia or even time loss. Such data should be used for assessment and can point towards the diagnosis (1). In the author's experience, despite chaotic presentations, most MPD patients relate well and are likeable.

\section{ASSESSMENT TOOLS AND INSTRUMENTS}

\section{Hypnosis}

Hypnotizability has been documented as an attribute of and auto-hypnosis as a potential major factor of MPD (14). MPD patients rapidly and easily fall into hypnotic (trance) states, and have high scores on measures of hypnotizability 
$(28,29,30)$. During psychotherapy sessions it is common to observe patients entering what appear to be trance (hypnotic) states if only for brief periods or seconds.

Hypnosis has several applications in MPD which can be divided into therapeutic and diagnostic. The former uses include relief of anxiety and distress, enabling the patient to use self-hypnosis as a way of mastering internal chaos, retrieval of repressed historical information, and as a means to obtain abreactive experiences $(5,9)$. As a diagnostic tool hypnosis facilitates emergence of alters and personality fragments, and should generally be used only after a significant trust has developed between a patient and therapist.

Hypnosis is most propitiously used after other means of making the diagnosis have been tried and some groundwork has been established to prepare the patient for what can be a disconcerting or traumatic recognition (30). Hypnosis of MPD patients is generally safe and easy to induce but does require investigation into whether the patient has experienced formal hypnosis in the past, the quality of that experience, and an in-depth understanding of the patient's current feelings about hypnosis. As with any diagnostic or therapeutic procedure, clearly articulated objectives will ensure a successful outcome. Previous negative experiences, current misgivings or fears require additional caution and postponement. Hypnosis is not without untoward effects. There are rare patients who become extremely distraught or hysterical following induction. As a rule, early in treatment the most important principle is the continuity of a therapeutic alliance and ensuring the patient's sense of security. In any event, initially, hypnosis should be used cautiously.

\section{Drug Facilitated Interviews}

The use of medication, either sodium amytal or sodium pentothal, in diagnostic interviews has been described by Putnam (9). Medications accomplish many of the same goals as hypnosis but with the addition of side effects such as sedation. The restraints and precautions mentioned above for hypnosis, also apply to drug facilitated interviews. Except for the patient who refuses, hypnosis should probably be the procedure of choice.

\section{Self-Report Instruments}

The Dissociative Experiences Scale (DES) is a twenty-eight question self-report screening instrument that rates dissociative symptoms and experiences (31). It is most efficiently used when given to those patients with suspected dissociative symptoms or a history of abuse. In the newly revised version (32), the patient indicates agreement by circling a percentage from $0 \%$ (not at all) to $100 \%$ (all the time). Scoring is calculated by taking the sums of the 28 scores and averaging. The operating characteristics of the test have been defined using a cutoff of 30 to screen for cases of dissociation, giving a sensitivity and specificity of $80 \%$ (33). Patients with MPD generally score above 40 , but a high percentage of patients with PTSD and other dissociative disorders score higher than 30. A positive score demarcates the need to pursue a more thorough evaluation. 


\section{Structured Interviews}

The Dissociative Disorders Interview Schedule (DDIS) and the Structured Clinical Interview for Dissociative Disorders (SCID-D) are two assessment tools that can be used to clarify the diagnosis. Both are time consuming, requiring from 45 minutes to three hours to complete, and generally are used in research settings rather than clinically (9).

The DDIS which contains 131 items was developed for the DSM-III. It diagnoses dissociative disorders, somatization disorders, major depression, and borderline personality disorder and asks about childhood sexual abuse. It is reported to have a high sensitivity and specificity for diagnosing MPD (34).

The SCID-D requires special training, is extensive and time consuming, but is highly sensitive to MPD and able to detect all five Dissociative Disorders (35).

\section{Biological Studies}

There are no biological tests that aid in making the diagnosis of MPD. Despite a higher than expected frequency of abnormal EEGs with non-specific changes (36), EEGs are of little utility except in differentiating the case of a patient suspected with partial complex seizures from MPD. Drug screens can be very helpful in identifying comorbid substance abuse and the occasional patient whose presentation is confused with a dissociative disorder.

\section{COMORBIDITY}

Comorbid diagnoses exist alongside the primary identified diagnosis, and sometimes the two nearly overlap (i.e., MPD and PTSD). Affective disorders frequently plague patients with MPD and are principal instigators of presentation to the mental health system $(9,37)$, but do not typically confuse clinicians per se; the exception to this generalization is bipolar disorder (38). Besides affective disorders, the most common comorbid diagnoses are anxiety disorders, substance abuse and dependence, eating disorders, and personality disorders. Most experts believe that MPD should be treated as a superordinate diagnosis that has a plethora of possible presentations $(1,9,15)$. Any psychiatric diagnosis may co-exist with MPD (23), but practically speaking, the list is finite.

\section{DIFFERENTIAL DIAGNOSIS}

What is most striking about the differential diagnosis for MPD is the preponderance of psychotic spectrum disorders in an illness that generally does not exhibit true psychotic symptoms (see table 2). This distinction has to do with the protean nature of MPD, but is also a reflection of lack of training on the part of clinicians who are not accustomed to conducting an interview for investigating the diagnosis. 
TABLE 2.

Differential Diagnosis of MPD

\begin{tabular}{l}
\hline Dissociative Disorders \\
Psychogenic amnesia \\
Psychogenic fugue \\
Dissociative disorder NOS \\
Depersonalization disorder \\
Organicity \\
Temporal lobe epilepsy \\
Alcohol Abuse and Dependence \\
Substance Abuse and Dependence \\
Psychotic Disorders \\
Schizophrenia \\
Schizoaffective disorder \\
Brief Reactive Psychosis \\
Delusional disorder \\
Mood Disorders \\
Major Depression with psychotic features \\
Bipolar disorders \\
Personality Disorders \\
Borderline personality disorder \\
Mixed Personality disorder \\
Malingering and Factitious disorder \\
Possession States \\
\hline
\end{tabular}

\section{CONCLUSION}

As an adaption to sustained sexual, physical, emotional abuse or neglect MPD has as a consequence for the adult profound negative intrapsychic and interpersonal effects. The expression of these effects often masquerades as other psychiatric illness. MPD as a polysymptomatic, pleiomorphic, and "hidden" condition is not necessarily easy to detect and is frequently denied by patients and overlooked by clinicians. Because of the variability of its symptomatic expression it requires patience on the part of the clinician to diagnose, but the rewards are significant if diagnosis leads to proper treatment disposition.

Questions pertaining to the non-specific symptoms of MPD must now be asked of all patients during the typical psychiatric interview: blackouts, history of sexual and physical abuse, and PTSD symptoms. Other symptoms require additional questioning to dissect out the diagnosis: auditory hallucinations, Schneiderian symptoms, amnesia, and behavioral changes. No symptoms are pathognomonic, and it is not always possible to make a conclusive diagnosis early in treatment, but the presence of a couple of symptoms should raise the clinician's index of suspicion. The identification or emergence of alters comes very close to a definitive diagnosis of MPD. In those cases that are suggestive but not definitive there are now several instruments that can facilitate in the detection of dissociative processes.

While the focus has been on the psychiatric interview and the symptom expression of MPD in adults, the importance of diagnosis in children, who are usually 
easier to treat (15), should be emphasized. For many cases MPD can be prevented altogether by effective child protection interventions. When child abuse has already occurred, long term treatment by experienced clinicians can ameliorate dissociative pathology. Prevention and attenuation of traumatic stress disorders, MPD being a very severe form, are crucial frontiers of preventive psychiatric medicine.

\section{ACKNOWLEDGEMENT}

The author would like to thank Kathy Reilly, M.A. and Kathy Pajer, M.D., M.P.H. for their helpful comments and suggestions.

\section{REFERENCES}

1. Loewenstein RJ: An Office Mental Status Examination for Complex Chronic Dissociative Symptoms and Multiple Personality Disorder, in Psychiatric Clinics of North America. Edited by Loewenstein RJ. W. B. Saunders Company, 1991

2. Ross CA: Epidemiology of Multiple Personality and Dissociation, in Psychiatric Clinics of North America. Edited by Loewenstein RJ. W. B. Saunders Company, 1991

3. Kluft RP: An Update on Multiple Personality Disorder, Hospital and Community Psychiatry 1988; 38(4):363-373

4. Spiegel D: Multiple Personality as a Post-Traumatic Stress Disorder, in Psychiatric Clinics of North America. Edited by Braun BG. W. B. Saunders Company, 1984

5. Kluft RP: The Dissociative Disorders, in Textbook of Psychiatry. Edited by Talbott JA, Hales RE, and Yudofsky SC. Washington, D.C., American Psychiatric Press, 1988

6. Coons PM: The Differential Diagnosis of Multiple Personality Disorder, in Psychiatric Clinics of North America. Edited by Braun BG. W. B. Saunders Company, 1984

7. American Psychiatric Association: Diagnostic and Statistical Manual of Mental Disorders, (Third Edition, Revised). Washington, D.C., American Psychiatric Association, 1987

8. Kluft RP: Clinical Presentation of Multiple Personality Disorder, in Psychiatric Clinics of North America. Edited by Loewenstein RJ. W. B. Saunders Company, 1991

9. Putnam FW: The Diagnosis and Treatment of Multiple Personality Disorder. New York, Guilford Press, 1989

10. Ross CA: Multiple Personality Disorder: Diagnosis, Clinical Features and Treatment. New York, John Wiley \& Sons, 1989

11. Franklin J: Diagnosis of Covert and Subtle Forms of Multiple Personality Disorder. Dissociation 1988; 1(2):27-33

12. Braun BG: The BASK Model of Dissociation. Dissociation 1988; 1:4-23

13. Watkins JG, Watkins HH: Ego-States and hidden observers. Journal of Altered States of Consciousness 1980; 5:3-18

14. Spiegel D, Cardena E: Disintegrated Experience: The Dissociative Disorders Revisited. Journal of Abnormal Psychology 1991; 100(3), 366-378

15. Kluft RP: Multiple Personality Disorder, in Review of Psychiatry, Volume 10. Edited by Tasman A and Goldfinger SM. American Psychiatric Press, 1991

16. DSM-IV Options Book: Work In Progress. Task Force on DSM-IV, American Psychiatric Association, Washington, D.C., 1991

17. Coons PM, Bowman ES, Kluft RP, Milstein L: The Cross-Cultural Occurrence of MPD: Additional Cases from a Recent Survey. Dissociation 1991; 6(3):124-128 
18. World Health Organization: The ICD-10 Classification of Mental and Behavioral Disorders. World Health Organization, Geneva 1992

19. Wilbur C: The Effect of Child Abuse on the Psyche, in Childhood Antecedents of Multiple Personality Disorder. Edited by Kluft RP. Washington, D.C., APA Press 1985

20. Goodwin J: Credibility Problems in Multiple Personality Disorder Patients and Abused Children, in Childhood Antecedents of Multiple Personality Disorder. Edited by Kluft RP. Washington, D.C., APA Press 1985

21. Briere J: Therapy for adults Molested as Children: Beyond Survival. New York, Springer Publishing Company 1989

22. Goodwin J, Cheeves K, Connell V: Defining a Syndrome of Severe Symptoms in Survivors of Severe Incestuous Abuse. Dissociation 1988; 1(4):11-16

23. Braun BG: Multiplicity: Form, Function and Phenomenon, 1991; adapted from Multiplicity: Form, Function and Phenomenon, Associated Mental Health Services, Chicago, 1984, 1987

24. Kluft RP: First-Rank Symptoms as a Diagnostic Clue to Multiple Personality Disorder. Am J Psychiatry 1987; 144:293-298

25. Ross CA, Joshi S: Schneiderian Symptoms in the General Population. Presented at the 8th International Conference on Multiple Personality/Dissociative States, Chicago, IL, November 1991

26. Loewenstein RJ: Psychogenic Amnesia and Psychogenic Fugue: A Comprehensive Review, in Review of Psychiatry, Volume 10. Edited by Tasman A and Goldfinger SM. American Psychiatric Press, 1991

27. Kopelman MD: Amnesia: Organic and Psychogenic. British Journal of Psychiatry 1987; 150:428-442

28. Spiegel D: Dissociation and Trauma, in Review of Psychiatry, volume 10. Edited by Tasman A and Goldfinger SM. American Psychiatric Press, 1991

29. Bliss E: Multiple Personality, Allied Disorders and Hypnosis. Oxford University Press, 1986

30. Braun BG: Uses of Hypnosis with Multiple Personality. Psychiatric Annals 1984; 14(1): $34-40$

31. Bernstein EM, Putnam FW: Development, Reliability, and validity of a Dissociation Scale. The Journal of Nervous and Mental Disease 1986; 174(12):727-735

32. Carlson EB, Putnam FW: An Update on the Dissociative Experiences Scale. Dissociation 1993; 6(1):16-27

33. Carlson EB, Putnam FW, Ross CA, Torem M, Coons P, Dill DL, Loewenstein RJ, Braun BG: Validity of the Dissociative Experiences Scale in Screening for Multiple Personality Disorder: A Multicenter Study. Am J Psychiatry 1993, 150:1030-1036.

34. Ross CA, Heber S, Norton GR, Anderson D, Anderson A, Barchet P: The Dissociative Disorders Interview Schedule: A Structured Interview. Dissociation 1989; 2(1):169-189

35. Steinberg M, Rounsaville B, Cicchetti D: Detection of Dissociative Disorders in Psychiatric Patients by a screening Instrument and a Structured Diagnostic Interview. Am J of Psychiatry 1991; 148:1050-1054

36. Devinsky O, Putnam F, Grafman J, Bromfield E, Theodore WH: Dissociative states and epilepsy. Neurology 1989; 39:835-840

37. Putnam FW, Guroff JJ, Silberman EK, Barban L, Post RM: The Clinical Phenomenology of Multiple Personality Disorder: Review of 100 Recent Cases. J Clin Psychiatry 1986; 47:285-293

38. Alarcon RD: Pseudomultiplicity a Clinical Manifestation of Rapid Cycling Affective Disorder in Borderline Personality? Annals of Clinical Psychiatry 1990; 2:127-133 